\title{
Colitis ulcerosa: a propósito de un caso
}

\author{
MS. Montoro Romero ${ }^{a}$, B. Juanes de Toledo ${ }^{b}$ E. Medina Benítez, \\ Y. Rodríguez Gild, A. Ballén Barragán ${ }^{d}$. \\ 'MIR-Pediatría. Hospital Universitario 12 de Octubre. Madrid. España. \\ ${ }^{b}$ Pediatra. CS El Espinillo. Madrid. España. \\ cPediatra. Servicio de Gastroenterología Infantil. Hospital Universitario 12 de Octubre. Madrid. España. \\ ¿Servicio de Anatomía Patológica. Hospital Universitario I 2 de Octubre. Madrid. España.
}

\section{Resumen}

La incidencia de la enfermedad inflamatoria intestinal ha experimentado un aumento en los últimos años, y los recientes avances aparecidos, tanto en su diagnóstico como en su tratamiento, obligan a reenfocar el problema desde Atención Primaria. Por eso nos ha parecido oportuno presentar un caso reciente de colitis ulcerosa atendido en nuestro centro.

Palabras clave: Enfermedad inflamatoria intestinal. Colitis ulcerosa. Atención Primaria.

\section{Ulcerative colitis: a case report}

\section{Abstract}

The incidence of inflammatory bowel disease has increased in the last years. Recent advances that have appeared in diagnosis and treatment make necessary to refocus the problem from primary care. So we felt appropriate to present a recent case of ulcerative colitis treated at our primary health care centre.

Key words: Inflammatory Bowel Disease. Ulcerative colitis. Primary Health Care.

\section{Introducción}

Cuando hablamos de enfermedad inflamatoria intestinal (EII) nos estamos refiriendo a un grupo de trastornos inflamatorios crónicos, de etiología desconocida y de carácter sistémico, que afectan fundamentalmente al tubo di- gestivo, y que cursan en forma de reagudizaciones y remisiones. Tanto la enfermedad de Crohn (EC) como la colitis ulcerosa (CU) han aumentado su incidencia en los últimos años, debutando uno de cada tres o cuatro casos durante la edad pediátrica'. La implicación del

Blanca Juanes de Toledo, blanca.juanesdetoledo@gmail.com

Los autores declaran no presentar conflictos de intereses en relación con la preparación y publicación de este artículo. 
pediatra de Atención Primaria (AP) tanto en la orientación diagnóstica precoz como en el manejo posterior de estos pacientes crónicos, intentando preservar un crecimiento y desarrollo óptimos $^{2,3}$, es de suma importancia.

\section{Caso clínico}

Varón de 11 años de edad, controlado en la consulta de AP por un cuadro de diarrea y pérdida de peso no cuantificada de un mes de evolución. Refiere deposiciones líquidas y explosivas, en número de 5-10 al día, con sangre roja y moco sin pus, que no ceden con el ayuno y le despiertan por la noche. Este cuadro no ha mejorado con suero de rehidratación oral y probióticos. No refiere antecedentes personales ni familiares de interés, viajes recientes ni ambiente epidémico familiar. El paciente es remitido a su hospital de referencia, iniciándose estudio en el Servicio de Gastroenterología Infantil.

Exploración física: peso de $60 \mathrm{~kg}$ (> P97); talla de $161 \mathrm{~cm}$ (> P97); TA 123/85 mm Hg; temperatura $36,7{ }^{\circ} \mathrm{C}$; frecuencia cardiaca $82 \mathrm{lpm}$. Buen estado nutricional; buen estado general; normocoloreado, bien hidratado y nutrido. Sin exantemas ni petequias. Cabeza y cuello: sin masas cervicales. Auscultación cardiaca y pulmonar: normal.
Abdomen: blando y depresible, doloroso a la palpación profunda a nivel periumbilical y del flanco izquierdo; sin hepatoesplenomegalia. Inspección anal: eritema perianal sin fisuras. Resto de la exploración por aparatos normal. Analítica: leucocitos 12 900/ $\mu \mathrm{l}(70,7 \%$ neutrófilos); hemoglobina $13,9 \mathrm{~g} / \mathrm{dl}$; hematocrito 41,6\%; plaquetas $375000 / \mu l$; glucosa $99 \mathrm{mg} / \mathrm{dl}$; albúmina 3,88 g/l; sodio $139 \mathrm{mEq} /$; creatinina 0,64 mg/dl; hierro $29 \mathrm{mg} / \mathrm{dl}$; transferrina $218 \mathrm{mg} / \mathrm{dl}_{\text {; }}$ ferritina $66 \mathrm{ng} / \mathrm{ml}$; TIBC $307 \mu \mathrm{g} / \mathrm{dl}_{\text {; }}$ saturación de la transferrina 9\%; IgC $1130 \mathrm{ng} / \mathrm{ml}$, IgA $125 \mathrm{ng} / \mathrm{ml}$, IgM $82 \mathrm{ng} / \mathrm{ml}$; anticuerpos IgA-antigliadina negativos; anticuerpos IgA-antitransglutaminasa negativos; proteína $C$ reactiva $4,66 \mathrm{mg} / \mathrm{dl}$.

Evolución: al segundo día del ingreso presenta pico febril de $39^{\circ} \mathrm{C}$. Estudio microbiológico: hemocultivos negativos; coprocultivo, parásitos, toxina de Clostridium difficile y antígeno de rotavirus en heces: negativo. Serologías negativas para virus de las hepatitis A, B y $C$, citomegalovirus y virus Epstein Barr.

Se realiza una ecografía abdominal, que se informa como engrosamiento mural de colon transverso y colon izquierdo, con cambios inflamatorios de grasa adyacente y presencia de adenopatías locorregionales. Se complementa 
con una resonancia magnética (RM) que confirma los hallazgos ecográficos, sin encontrar afectación del intestino delgado.

Se realiza una colonoscopia, introduciendo el endoscopio hasta lo que parece corresponder al ángulo esplénico. Se observa afectación continua con una mucosa muy friable al roce y se toman muestras a 100, 70, 55, y $45 \mathrm{~cm}$ del margen anal. A la retirada del endoscopio se aprecia afectación de la mucosa rectal de aspecto petequial y sin otras lesiones focales.
En todas las muestras para estudio histopatológico se observaron lesiones que eran continuas y afectaban a todos los fragmentos. Las lesiones correspondían a erosiones con reepitelización de forma continua (figura 1). Había distorsión de la arquitectura que demostraba afectación crónica. Además, el grado de actividad en el momento de la biopsia era de moderado a intenso, con presencia de microabcesos y criptitis frecuentes (figura 2). No se observaron granulomas. No había imágenes de ulceración transmural, y las lesiones estaban limita-

Figura 1. Tinción HE (200x): áreas de erosión y reepitelización que afectaban de forma continua a toda la mucosa desde el recto hasta el colon derecho. Se observa actividad y distorsión de la arquitectura tisular.

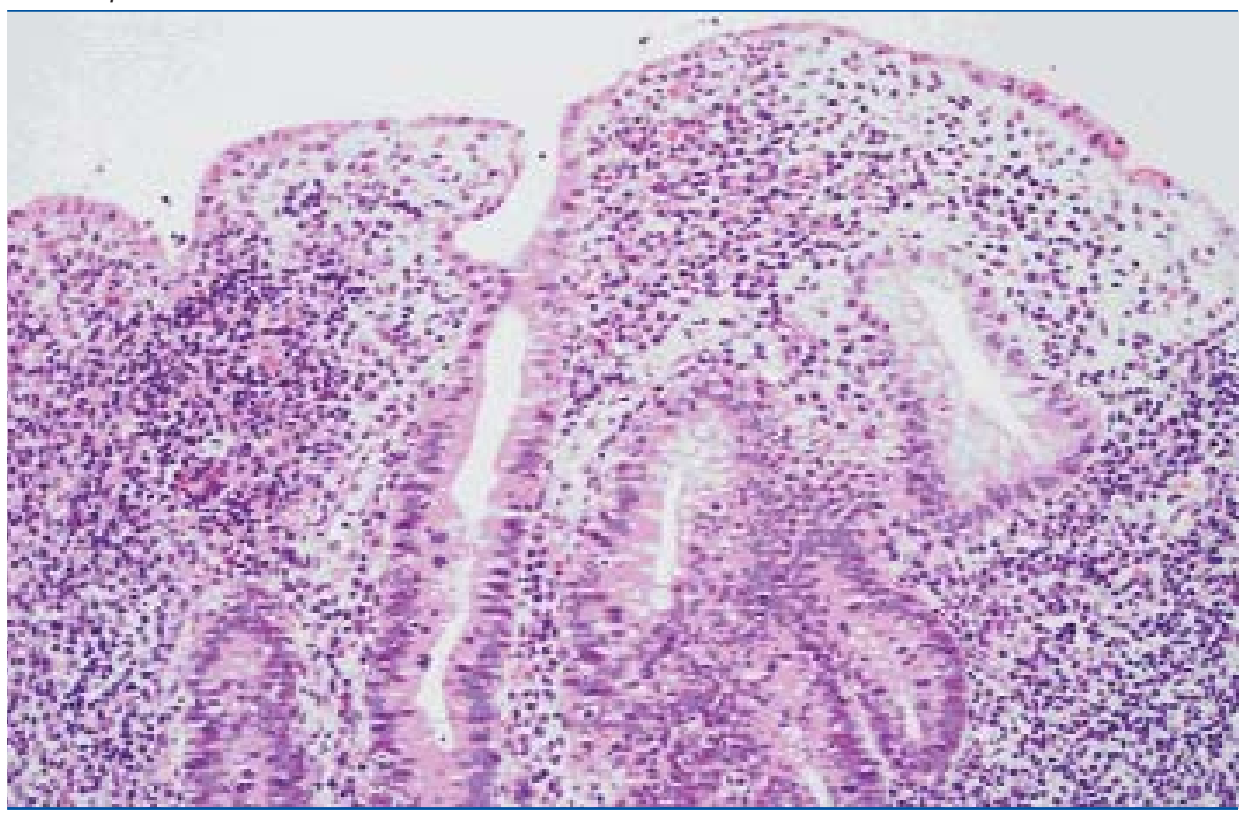


Figura 2. Tinción HE (400x): imagen de gran aumento con permeación de las criptas por neutrófilos formando criptitis y microabscesos.

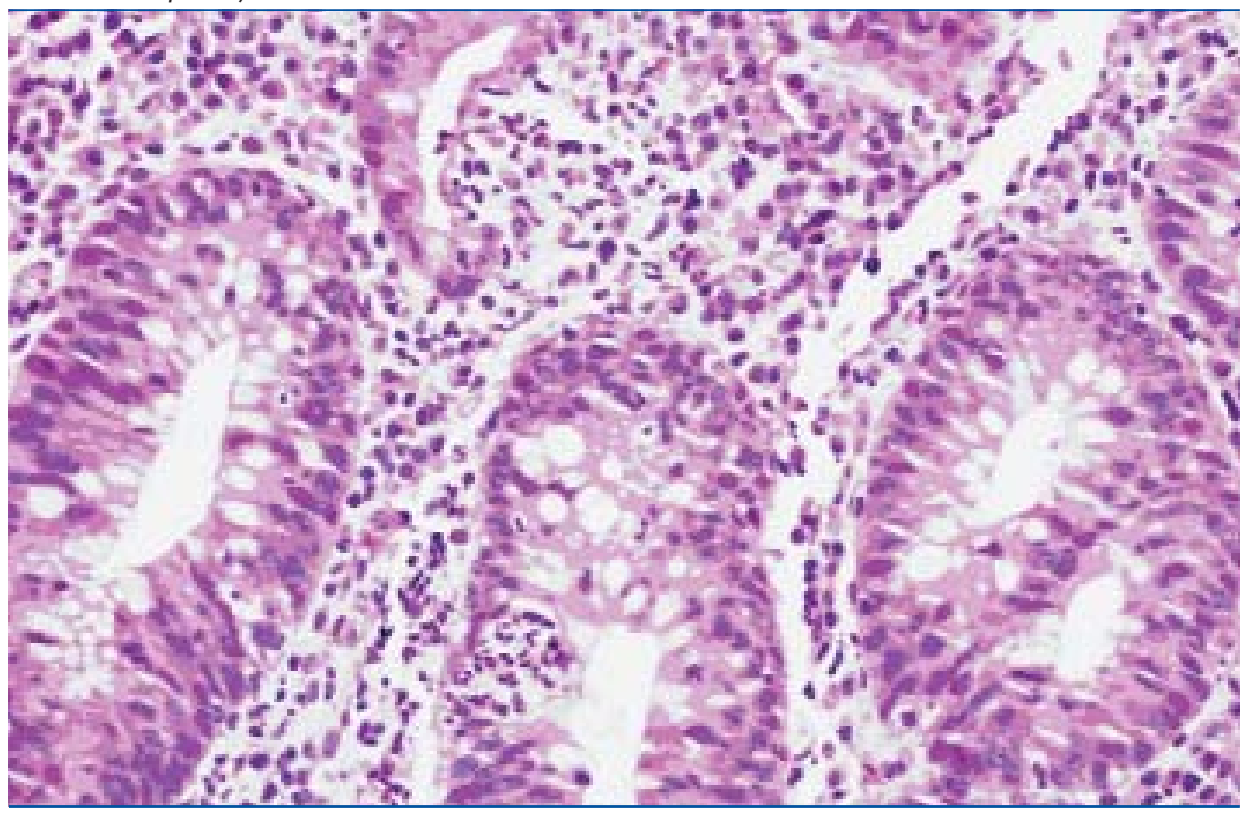

das a la mucosa (epitelio y corion), con escasa afectación de la submucosa.

Ante los hallazgos de la colonoscopia y la afectación del estado general, se decide comenzar tratamiento con corticoides IV a 60 mg/día (durante cuatro días para pasar a vía oral posteriormente) y mesalazina oral $1 \mathrm{~g} / 8$ horas.

Tras la mejoría, es dado de alta hospitalaria a los diez días del ingreso con el diagnóstico de posible colitis ulcerosa y tratamiento ambulatorio con mesalazina (1 g/8 horas, vía oral), metilprednisolona (40 mg/día, vía oral) y omeprazol (20 mg/día, vía oral).

\section{Discusión}

La incidencia de la Ell ha aumentado en los últimos años y hasta un 30\% de los casos debuta en la infancia. Dentro de esta, la CU se puede definir como un proceso inflamatorio crónico de la mucosa del colon de forma continua desde el recto, y en una extensión variable; biopsia sin granulomas, y curso con reagudizaciones y remisiones. La prevalencia de la CU en España es de 70-150/100 000 habitantes, y de la EC de 60-120/100 000 habitantes, con una incidencia de 8/100 000 y 5,5/100 000 habitantes, respectivamen$t^{4}$. Es por ello una patología que debe- 
mos tener en cuenta en el diagnóstico diferencial de la diarrea crónica en nuestras consultas, sobre todo en adolescentes.

Siguiendo los criterios de Oporto ${ }^{5}$, el diagnóstico de la Ell debe contemplar la localización de la enfermedad, su extensión en el tracto gastrointestinal, y la actividad del proceso inflamatorio a lo largo de su evolución:

- El cuadro clínico persistente ( $\geq 4$ semanas) o recurrente ( $\geq 2$ episodios en seis meses) de diarrea sanguinolenta, dolor abdominal, y pérdida de peso en un escolar o adolescente, debe hacer pensar en Ell.

- El diagnóstico diferencial obliga a realizar cribado celíaco, descartar causa infecciosa mediante coprocultivo y parásitos, y tuberculosis intestinal en población de riesgo.

- El diagnóstico de la Ell se basa en los hallazgos endoscópicos e histológicos. La capsuloendoscopia facilita el estudio del intestino delgado.
- En cuanto a la actividad de la enfermedad, sigue siendo válido el índice de Truelove-Witts que combina criterios clínicos y analíticos de manera objetiva (tabla 1), y se debe valorar de forma periódica en la consulta de AP.

El tratamiento de la $\mathrm{El}^{2}$ se ha basado clásicamente en la combinación de aminosalicilatos, corticoides y antibióticos, junto con algunos inmunosupresores y las nuevas terapias biológicas. Dentro de los aminosalicilatos, están los inhibidores de la lipooxigenasa (5-ASA y mesalazina). Tienen un papel antiinflamatorio $y$ anticarcinogénico, pero pueden presentar efectos secundarios hasta en un $40 \%$ de los casos. Entre los corticoides, la prednisona, la metilprednisolona y recientemente la budesonida, tanto oral como en enemas locales (con menos efectos secundarios), inducen mejoría clínica de un $60-90 \%$ de los pacientes, aunque no histológica. Se ha usado el metronidazol con

\begin{tabular}{|c|c|c|c|c|}
\hline \multicolumn{2}{|c|}{ Puntuación } & 1 & 2 & 3 \\
\hline \multicolumn{2}{|c|}{ Número de deposiciones/día } & $<4$ & $4-6$ & $>6$ \\
\hline \multicolumn{2}{|c|}{ Sangre en heces } & No & Escasa & Abundante \\
\hline \multicolumn{2}{|c|}{ Temperatura axilar $\left({ }^{\circ} \mathrm{C}\right)$} & $<37$ & $37-37,5$ & $>37,5$ \\
\hline \multicolumn{2}{|c|}{ Frecuencia cardiaca (lpm) } & $<80$ & $80-90$ & $>90$ \\
\hline \multirow[t]{2}{*}{ Hemoglobina (g/dl) } & Varones & $>14$ & $10-14$ & $<10$ \\
\hline & Mujeres & $>12$ & $9-12$ & $<9$ \\
\hline \multicolumn{2}{|c|}{ Velocidad de sedimentación globular } & $<20$ & $20-30$ & $>30$ \\
\hline
\end{tabular}


el fin de disminuir la concentración de bacterias, inflamación de la mucosa y respuesta inmunitaria celular. Los inmunomoduladores (AZA-6MP) se utilizan en EC y pancolitis severas desde el momento del diagnóstico, junto con nutrición enteral exclusiva. Hay que sopesar riesgo-beneficio ante la posible aparición de mielosupresión o procesos linfoproliferativos. Dentro de las terapias biológicas, el infliximab y el adalimumab (anticuerpos monoclonales antifactor de necrosis tumoral alfa) han cambiado el tratamiento de la Ell y se utiliza en la CU moderada-grave. Posiblemente sea utilizable como primera línea de tratamiento por su alta efectividad al inducir remisión clínica e histológica rápida en el 75\% de los enfermos, disminuir las recaídas, el uso de corticoides, evitando pérdida de masa ósea y las secuelas de la enfermedad.

En definitiva, el pediatra de AP puede sospechar el diagnóstico, controlar el desarrollo psicológico y nutricional del niño con Ell y la posible utilización de terapias alternativas $s^{6} ; \mathrm{y}$ valorar la actividad de la enfermedad ante la aparición de nuevos brotes que precisen intensificación o cambios en el esquema nutricional y/o terapéutico.

\section{Bibliografía}

1. Quero Acosta L, Argüelles Martín F, Artieda Osealde M. Nuevas herramientas no invasivas en el diagnóstico y seguimiento de la Enfermedad Inflamatoria intestinal. Utilidad de las tradicionales. Pediatr Integral. 2007;11(2):13344.

2. Suárez L, Agrimbau JD, Fuentes D. Enfermedad inflamatoria intestinal: Situación actual de las alternativas terapéuticas. Colomb Med. 2007;38 (Supl 1):34-40.

3. Vitoria Cormenzana JC, Pérez Estévez E. Actualización en patología del intestino delgado: enfermedad celiaca y enfermedad inflamatoria intestinal. En: AEPap (ed.). Curso de Actualización en Pediatría 2006. Madrid: Exlibris Ediciones; 2006. p. 91-9 [consultado el 28/07/2010].
Disponible en www.aepap.org/congresos/cur so2006.php?pdf=celiaca_eii.pdf

4. García Betanzos R. Enfermedad Inflamatoria Intestinal. Guía Clínica. Fisterra: Atención Primaria de Salud en la Red [consultado el 28/07/2010]. Disponible en www.fisterra.com/ guias2/eii.asp\#diferencial

5. IBD Working Group of the European Society for Paediatric Gastroenterology, Hepatology and Nutrition (ESPGHAN). Inflammatory Bowel Disease in Children and Adolescents: Recommendations for Diagnosis-The Porto Criteria. J Pediatr Gastroenterol Nutr. 2005;41:1-7.

6. Juanes de Toledo B, Aparicio Rodrigo M. Casi la mitad de los niños con enfermedad inflamatoria intestinal utilizan medicinas alternativas. Evid Pediatr. 2009;5:46 [consultado el 28/07/ 2010]. Disponible en www.evidenciasenpedia tria.es 